\title{
MOUFANG AFFINE BUILDINGS HAVE MOUFANG SPHERICAL BUILDINGS AT INFINITY
}

\author{
by H. VAN MALDEGHEM $\dagger$ and K. VAN STEEN
}

(Received 23 March, 1995)

\begin{abstract}
We show in a direct and elementary way that the spherical building at infinity of every rank 3 affine building which satisfies Tits' Moufang condition, is itself a Moufang building. This result is also true for higher rank affine buildings by Tits' classification [4].

1. Introduction. The Moufang condition for-not necessarily spherical-buildings was introduced by Tits [5]. It generalizes the usual Moufang condition for spherical buildings, see Tits [3], which on its turn was a generalization of the Moufang condition for projective planes. The Moufang condition seems to be the most natural condition under which a classification of certain classes of buildings is possible. For spherical buildings of rank $\geq 3$, and for affine building of rank $\geq 4$, this is trivially true for all those buildings are classified without any supplementary condition. For spherical buildings of rank 2, Tits [3] announces such classification and partial results have been published. There seems to be no further explicit classification of Moufang affine buildings of rank 3 in the literature. In this short note, we show that such a classification can be reduced to checking the Moufang property in the "known classical buildings". Our method uses the building at infinity of the affine building. The definition of Moufang affine building does not imply ipso facto that the building at infinity also satisfies the Moufang condition. We will show that this is however a consequence, using elementary techniques. So our main result reads:
\end{abstract}

MaIN RESULT. The building at infinity of an irreducible Moufang rank 3 affine building is a Moufang rank 2 spherical building.

COROLLARY. The irreducible Moufang rank 3 affine buildings are amongst the affine buildings arising from an algebraic group of relative rank 2 defined over a field with discrete valuation (with respect to which the field is complete), which is invariant under the field involution that is possibly needed to define the group; also, the local field has equal characteristic.

2. Preliminaries. In this paper, we take the original viewpoint of Tits [2]. So buildings are thick simplicial chamber complexes endowed with a set of thin subcomplexes (these subcomplexes are called apartments) such that every two simplices are contained in a common apartment, and such that for any pair of apartments $\left(\Sigma, \Sigma^{\prime}\right)$, there exists an isomorphism $\theta: \Sigma \rightarrow \Sigma^{\prime}$ fixing every simplex contained in the intersection of $\Sigma$ and $\Sigma^{\prime}$. It turns out that the apartments of a building $\Delta$ are Coxeter complexes and that the Coxeter diagram of this complex is the same as the Buekenhout diagram of the geometry associated to $\Delta$ (see Tits [2]). In this paper, we are concerned with the (irreducible) rank 3 affine cases, i.e. the types $\tilde{A}_{2}, \tilde{C}_{2}$ and $\tilde{G}_{2}$. We briefly describe the Coxeter complexes of each of these types.

$\dagger$ This author is a Senior Research Associate at the Belgian National Fund for Scientific Research.

Glasgow Math. J. 39 (1997) 237-241. 
$\left(\tilde{A}_{2}\right) \quad$ Consider the triangulation of the real euclidian plane $\mathbb{E}$ with equilateral triangles. The vertices of the Coxeter complex are the vertices of the triangles, the edges form simplices of dimension 1 and triangles itself define simplices of dimension 2. This Coxeter complex can also be defined as the barycentric subdivision of the tiling of $\mathbb{E}$ into regular hexagons.

$\left(\bar{C}_{2}\right)$ Here we triangulate $\mathbb{E}$ with isosceles right triangles. Or we consider the barycentric subdivision of the tiling of $\mathbb{E}$ into regular octagons and squares.

$\left(\widetilde{G}_{2}\right) \quad$ Here we triangulate $\mathbb{E}$ with right triangles having an angle of $30^{\circ}$. Or we consider the barycentric subdivision of the tiling of $\mathbb{E}$ into regular 12-gons, hexagons and squares.

A special vertex in these complexes is a vertex lying in a maximal number of chambers (these numbers are respectively 6,8 and 12). A ray is the set of vertices lying on a half line in $\mathbb{E}$ starting at a special vertex and containing edges of triangles of the respective triangulation. A sector is the set of all vertices belonging to the convex closure in $\mathbb{E}$ of two rays starting at the same special vertex and forming a minimal angle (i.e. $60^{\circ}$, $45^{\circ}$ and $30^{\circ}$ in the three respective cases). A special vertex, a ray or a sector in a rank 3 affine building $\Delta$ is a special vertex, a ray of a sector respectively in some apartment of $\Delta$. Also, a panel is a simplex consisting of two vertices.

Let $\Delta$ be a rank 3 affine building. Suppose that we endow $\Delta$ with a maximal set of apartments (this is always possible by Tits [4]). Note that the metric of $\mathbb{E}$ induces a metric in $\Delta$. The relation $R:$ : . . is at bounded distance from..." is an equivalence relation in the set of all rays. We define the following simplicial complex $\Delta_{\infty}$. The vertices of $\Delta_{\infty}$ are the equivalence classes of rays with respect to $R$; the simplices of dimension 1 (also the chambers) are the pairs of such classes for which there exist respective representatives which lie in a common sector. It can be shown that $\Delta_{x}$ is a rank 2 spherical building and every automorphism of $\Delta$ also preserves the structure of $\Delta_{x}$ (see Tits [4]). Note that, by Tits [4], each equivalence class of rays has a (unique) representative starting at any special vertex.

The union of two rays in an apartment $\Sigma$ of a rank 3 affine building $\Delta$ which start from the same vertex and which form an angle of $180^{\circ}$ is called a wall; each wall $w$ in $\Sigma$ divides $\Sigma$ in two half apartments $\alpha$ and $-\alpha$ which share the vertices in $w$. We denote $w$ by $\partial \alpha$. Parallel walls are walls which lie on parallel lines in some apartment. Each half apartment is called a root. Let $\Phi$ be the set of roots in a given apartment $\Sigma$ of $\Delta$. Given $\alpha \in \Phi$, we denote, as above, by $-\alpha$ the complementary root in $\Sigma$ (so $\alpha$ and $-\alpha$ meet in the wall $\partial \alpha=\partial(-\alpha)$ ). For two roots $\alpha$ and $\beta$, we write, following Tits [5],

$$
[\alpha, \beta]=\{\gamma \in \Phi: \alpha \cap \beta \subseteq \gamma \text { and }(-\alpha) \cap(-\beta) \subseteq(-\gamma)\}
$$

We call $\Delta$ a pre-Moufang building if, for some apartment $\Sigma$, there is a family of automorphism groups $\left(U_{\alpha}\right)_{\alpha \in \Phi}$ (where $\Phi$ is defined as above) of $\Delta$ satisfying the following two conditions:

(pM1) For each $\alpha \in \Phi$ and each panel $p$ in $\alpha \cap(-\alpha)$, the group $U_{\alpha}$ fixes every vertex of $\alpha$ and acts transitively on the set of chambers containing $p$ and not contained in $\alpha$.

(pM2) If $\alpha, \beta \in \Phi$ and $\partial \alpha$ and $\partial \beta$ are not parallel, then the commutator $\left[U_{\alpha}, U_{\beta}\right]$ is contained in the group generated by all $U_{\gamma}$, where $\gamma \in[\alpha, \beta] \backslash\{\alpha, \beta\}$. 
A Moufang building satisfies two further properties (and stronger versions of both (pM1) and (pM2)), but we will not need these here. It suffices to remark that every Moufang rank 3 affine building is a pre-Moufang building, and that the Moufang condition requires that the transitive action in (pM1) is simply-transitive. This follows immediately from Tits [5]; see also Ronan [1].

Finally, we define a Moufang rank 2 building, as introduced by Tits $[2,3]$. Let $\Gamma$ be a rank two spherical building and let $\Sigma$ be some apartment in $\Gamma$. We can view $\Sigma$ as a $2 n$-path (connected vertices form a panel; such vertices are also called adjacent), for fixed $n \in \mathbb{N} \backslash\{0,1,2\}$ (and $\Gamma$ corresponds to a so-called generalized $n$-gon). If for each $n$-path $\left(x_{0}, x_{1}, \ldots, x_{n}\right)$ in $\Sigma$, the group of automorphisms of $\Gamma$ fixing all vertices of $\Gamma$ adjacent to one of $x_{1}, x_{2}, \ldots, x_{n-1}$ acts transitively on the set of vertices of $\Gamma$ adjacent to $x_{0}$ but distinct from $x_{1}$, then $\Gamma$ is called a Moufang rank 2 building.

3. Proof of the main result. From now on, we suppose that $\Delta$ is a pre-Moufang rank 3 affine building containing an apartment $\Sigma$ (with corresponding set of roots $\Phi$ ) and such that there exists a family of groups $\left(U_{\alpha}\right)_{\alpha \in \Phi}$ which satisfy (pM1) and (pM2). We consider the rank 2 spherical building $\Delta_{\infty}$ and we let $s$ be any special vertex in $\Sigma$. By the previous section, we may identify the vertices of $\Delta_{\infty}$ with the rays starting at $s$. It follows from Tits [4] that adjacent vertices correspond to rays forming a minimal angle.

The rays in $\Sigma$ starting in $s$ define a $2 n$-path $\Pi$ of $\Delta_{\infty}(s), n=3,4,6$ for respectively $\Delta$ of type $\tilde{A}_{2}, \tilde{C}_{2}, \tilde{G}_{2}$. Note that $\Pi$ does not depend on $s$. The rays starting in $s$ and corresponding to an $n$-path $\pi$ contained in $\Pi$ all lie in a unique well-defined root $\pi_{s}$ with $s \in \partial \pi_{s}$. Let $\pi=\left(x_{0}, x_{1}, \ldots, x_{n}\right)$, then we show that every element of $U_{n_{s}}$, for arbitrary $s$, fixes every vertex of $\Delta_{\infty}$ adjacent to $x_{1}$.

Indeed, let $r$ be the ray starting in $s$ and representing the vertex $x$ of $\Delta_{\infty}$ which is adjacent to $x_{1}$. Let $r_{i}$ be the ray starting in $s$ and representing $x_{i}$. Since $x$ is adjacent to $x_{1}$, the ray $r$ "leaves" the apartment $\Sigma$ at a vertex $s^{\prime}$ of $r_{0}$ or $r_{2}$ (i.e. the vertex $s^{\prime}$ is the common vertex of $r$ and $\Sigma$ at maximal distance from $s$ ). By considering the intersection of $\Sigma$ with a sector containing $r$ and $r_{1}$ (and noting that this intersection is convex in the sense of Tits [2]), one sees that $s^{\prime}$ is a special vertex (because it is contained in two rays forming a minimal angle).

From now on, the term "distance", denoted by $\delta$, applies to the natural distance in the adjacency graph of $\Delta$. We show by induction on $m$, that the vertex $v_{m}$ on $r$ at distance $m$ from $s^{\prime}$ and distance $m+\delta\left(s, s^{\prime}\right)$ from $s$ is fixed by every element $\theta$ of $U_{\pi_{i}}$. So let $\theta \in U_{\pi_{s}}$. In view of (pM1) the claim holds for $v_{0}=s^{\prime}$, i.e. for $m=0$. Now let $v_{m}$ be arbitrary on $r$ and suppose $\delta\left(s^{\prime}, v_{m}\right)=m>0$. Let $\pi^{\prime}$ be the $n$-path $\left(x_{1}, x_{2}, \ldots, x_{n}, x_{n+1}\right)$ (with $x_{n+1}$ in II). By (pM1), there exists $\theta^{\prime} \in U_{\pi^{\prime}}$, mapping $v_{1}$ in $\Sigma$. By the induction hypothesis, $v_{m}^{\theta^{\prime}}$ is fixed by $\theta$ (even if $\theta^{\prime}$ does not preserve $s$ ) and hence we obtain that $v_{m}^{\theta^{\prime} \theta \theta^{\prime-1}}=v_{m}$. But $(\mathrm{pM} 2)$ implies $\theta^{\prime} \theta \theta^{\prime-1}=\theta$, hence the claim. So we have shown that $\theta$ preserves $r$ and hence $x$.

Now suppose that $x$ is a vertex of $\Delta_{\infty}$ adjacent to $x_{2}$. In order to show that $\theta \in U_{\pi_{s}}$ fixes the ray $r$ representing $x$, we can copy the above proof up to the very last point, i.e. we obtain $v_{m}^{\theta^{\prime} \theta \theta^{\prime-1}}=v_{m}$, with $v_{m}$ defined similarly as before as the vertex in $\Delta$ at distance $m$ from the vertex $s^{\prime}\left(r\right.$ "leaves" $\Sigma$ at $\left.s^{\prime}\right)$ and distance $m+\delta\left(s, s^{\prime}\right)$ from $s$, and with

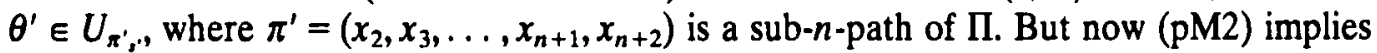
$\theta^{\prime} \theta \theta^{\prime-1} \in U_{\pi^{\prime \prime}} . \theta$, where $\pi^{\prime \prime}=\left(x_{1}, x_{2}, \ldots, x_{n}, x_{n+1}\right)$ and $s^{n}$ is the intersection of $\partial \pi_{s}$ and $\partial_{s}^{\prime}$. This notation includes the case where $s^{\prime \prime}$ is not a special vertex, and then one must 
put $U_{\pi_{*}^{*}}=\{1\}$. But in this case the result follows similarly as before. Hence, we may assume that $s^{\prime \prime}$ is a special vertex. Substituting $\pi^{\prime \prime}$ for $\pi$ and $s^{\prime \prime}$ for $s$ in the previous claim one sees that each element of $U_{\pi^{\prime \prime}}$, fixes $v_{n}$. Hence the result again follows.

Similarly, one shows that each element of $U_{n_{r}}$ fixes every vertex of $\Delta_{\infty}$ adjacent to $x_{i}$, $i \in\{1,2, \ldots, n-1\}$ (actually, by symmetry, there is only one case left: $(n, i)=(6,3)$ ).

Now we define $V_{\pi}$ to be the automorphism group of $\Delta_{x}$ generated by all $U_{\pi_{t}}$, for all special vertices $s$ in $\Sigma$. The group $V_{\pi}$ cannot be the right choice for $U_{\pi}$ since it certainly cannot act transitively on the set $S$ of vertices of $\Delta_{x}$ adjacent to $x_{0}$ and different from $x_{1}$. Indeed, $S$ has a higher cardinal number than $V_{\pi}$, e.g. if $\Delta$ is locally finite, then $V_{\pi}$ is countable and $S$ is not (that is because we are considering the maximal set of apartments for $\Delta$ ).

Let $s$ be any special vertex in $\Sigma$ and let $V_{\pi_{s}}$ be the automorphism group of $\Delta$ generated by all $U_{\pi^{\prime}}$, for all $s^{\prime}$ such that the root $\pi_{s^{\prime}}$ contains $s$ as a vertex. It is clear that $V_{\pi_{s}}$ preserves the sphere of radius $m$ in $\Delta$ centered at $s$, for all positive integers $m$. When restricting these spheres to vertices on rays starting in $s$, and by defining a new suitable-but obvious-adjacency relation, one sees easily that $\Delta_{x}$ can be identified with the inverse limit of all these restricted spheres. Now we denote by $\bar{U}_{n_{s}}$ the group of all automorphisms of $\Delta_{\infty}$ obtained by considering all possible inverse limits of automorphisms of these spheres induced by elements of $V_{\pi_{s}}$. Finally, we define $U_{\pi}$ as the group generated by all $\bar{U}_{\pi_{s}}$ for all special vertices $s$ in $\Sigma$. We show that $U_{\pi}$ acts transitively on $S$ (and from our proof, it will follow that $U_{\pi}$ is in fact the union of all $\bar{U}_{\pi_{s}}$ ).

Let $x_{-1} \in S$ be the vertex in $\Pi$ adjacent to $x_{0}$ and different from $x_{1}$. Let $x \in S$ be arbitrary. As before we consider a ray $r$ respectively $r_{i}$ representing $x$ respectively $x_{i}$ and starting at some special vertex in $\Sigma, i=-1,0$. Again the vertex $s$ where $r$ leaves $\Sigma$ is special and we can assume without loss of generality that $r, r_{0}$ and $r_{-1}$ all start in $s$. Denote by $v_{m}$ respectively $v_{-m}$ the vertex of $r$ respectively $r_{-1}$ at a distance $m$ from $s$. Condition (pM1) implies the existence of an element $\theta_{1} \in U_{\pi_{j}}$ mapping $v_{1}$ to $v_{-1}$. If $v_{k}, v_{k} \neq s$, is the special vertex of $r$ closest to $s$, then as before one sees by considering the intersection of $\Sigma$ with any sector containing $r$ and $r_{0}$ that $v_{k}^{\theta_{1}}=v_{-k}$. Let $\theta_{2} \in U_{\pi_{s-k}}$ be such that it maps $v_{k+1}^{\theta_{1}}$ to $v_{-k-1}$. It is now clear how to continue and to define $\theta_{l}$, for all positive integers $l$. It is also clear that the inverse limit of $\left(\theta_{1}, \theta_{1} \theta_{2}, \theta_{1} \theta_{2} \theta_{3}, \ldots\right)$ maps $r$ to $r_{-1}$. Hence the Main Result is proved.

Remarks. It is easily seen in $\Delta_{\infty}$ that $U_{\pi}$ acts simply-transitively on $S$. Hence $U_{\pi}$ is indeed the (non-disjoint) union of all $\bar{U}_{n_{s}}$ by the last paragraph of the proof.

The restricted spheres of radius $m$ that turn up above are in case of type $A_{2}$ Hjelmslev-planes of level $m$, see Van Maldeghem [6]. These geometries were used by Van Maldeghem in various papers to characterize affine buildings of type $\tilde{A}_{2}$ and $\tilde{C}_{2}$, for instance in terms of valuations on their spherical building at infinity [7].

If we endow $\Delta$ with a symmetric system of apartments (see Tits [4]), then our main result still holds since the building at infinity of such a system of apartments is a subbuilding of the one obtained from the full system of apartments.

4. Proof of the corollary. The corollary follows immediately from section 14 of Tits [4]. By Tits [2], this determines all irreducible rank 3 affine buildings. The assertion about the residue field follows directly from the fact that the root groups in $\Delta$ are subgroups of 
root groups in $\Delta_{\infty}$. Since for a Moufang rank 3 affine building, the root groups of $\Delta$ act simply-transitive on an "affine line" in the residue of a special vertex, the characteristics in question must be the same. However, it is not clear to us whether this condition is also sufficient to imply the Moufang condition. In fact, this is an open question for affine buildings of arbitrary rank.

\section{REFERENCES}

1. M. A. Ronan, Lectures on Buildings, Perspectives in Mathematics 7 (Academic Press, London 1989).

2. J. Tits, Buildings of spherical type and finite BN-pairs, Lecture Notes in Mathematics 386 (Springer-Verlag, 1974).

3. J. Tits, Classification of buildings of spherical type and Moufang polygons: a survey, Atti dei Convegni Lincei 17 (1976) 229-246.

4. J. Tits, Immeubles de type affine, in Buildings and the Geometry of Diagrams, Como 1984, Lecture Notes in Mathematics 1181 (Springer-Verlag, 1986), 159-190.

5. J. Tits, Uniqueness and presentation of Kac-Moody groups over fields, J. Algebra 105 (1987), 542-573.

6. H. Van Maldeghem, On projective Hjelmslev-planes of level n, Glasgow Math. J. 31 (1989), 257-261.

7. H. Van Maldeghem, Generalized polygons with valuation, Arch. Math. 53 (1989), 513-520.

Department of Pure Mathematics and Computer Algebra

UNIVERSITY OF GHENT

GalglaAN 2, B-3000 Gent, Belgium 